\title{
Shear-Improved Smagorinsky Model for Large-Eddy Simulation of Wall-Bounded Turbulent Flows
}

\author{
By E. Lévêque ${ }^{1}$, F. Toschi ${ }^{2,3}$, L. Shao ${ }^{4}$ and J.-P. Bertoglio ${ }^{4}$ \\ ${ }^{1}$ Laboratoire de Physique, CnRs, École normale supérieure de Lyon, France. \\ 2 Istituto per le Applicazioni del Calcolo, CNR, Viale del Policlinico 137, I-00161, Roma, Italy. \\ ${ }^{3}$ INFN, Sezione di Ferrara, Via G. Saragat 1, I-44100 Ferrara, Italy. \\ ${ }^{4}$ Laboratoire de Mécanique des Fluides et d'Acoustique, CNRS, École centrale de Lyon, France.
}

(Received 29 October 2018)

A shear-improved Smagorinsky model is introduced based on recent results concerning shear effects in wall-bounded turbulence by Toschi et al. (2000). The Smagorinsky eddyviscosity is modified as $\nu_{T}=\left(C_{s} \Delta\right)^{2}(|\bar{S}|-|\langle\bar{S}\rangle|)$ : the magnitude of the mean shear $|\langle\bar{S}\rangle|$ is subtracted to the magnitude of the instantaneous resolved strain-rate tensor $|\bar{S}|$; here $C_{S}$ is the standard Smagorinsky constant and $\Delta$ denotes the grid spacing. This subgrid-scale model is tested in large-eddy simulations of plane-channel flows at Reynolds numbers $R e_{\tau}=395$ and $R e_{\tau}=590$. First comparisons with the dynamic Smagorinsky model and direct numerical simulations, including mean velocity, turbulent kinetic energy and Reynolds stress profiles, are shown to be extremely satisfactory. The proposed model, in addition of being physically sound, has a low computational cost and possesses a high potentiality of generalization to more complex non-homogeneous turbulent flows.

\section{Introduction}

The prohibitive cost of direct numerical simulations (DNs) of turbulent engineering flows motivate the elaboration of simplified models, requiring less computation but still relevant (to some degree) for reproducing the large-scale dynamics (Deardorff 1970; Lesieur \& Metais 1996; Piomelli 1999; Sagaut 2001). In this context, the modeling near a solid boundary is of particular interest. A boundary affects the kinetics of the flow through different mechanisms; the most prominent is that related to the mean shear, which is extremal in the proximity of the boundary and responsible for the production of streamwise vortices and streaky structures. These fluid structures eventually detach from the boundary and sustain turbulence in the bulk (Perot \& Moin 1995). Thus, it is thought that understanding how the mean shear impacts on fluid motions is a key to improving the capabilities of numerical models of turbulent flows. Turbulence near a boundary is not homogeneous nor isotropic and therefore, the customary theoretical background for modeling the unresolved small-scale dynamics should be surpassed (Pope 2000). In the present work, we present a new subgrid-scale model of turbulence which may be viewed as an improvement over the popular Smagorinsky model in the presence of a mean shear. Our model originates from recent theoretical findings concerning shear effects in wall-bounded turbulence, see Toschi et al. (2000). We propose to subtract the magnitude of the mean shear from the resolved strain-rate tensor in the definition of the eddy-viscosity. As this will be discussed later, this simple improvement accounts for the non-isotropic nature of the flow near the boundary and at the same time, allows 
us to recover the standard Smagorinsky model in regions of (locally) homogeneous and isotropic turbulence. The general framework of the so-called large-eddy simulation (LES) of turbulent flows is now briefly recalled.

Roughly speaking, large-scale motions transport most of the kinetic energy of the flow. Their strength make them the most efficient carriers of conserved quantities (momentum, heat, mass, etc.). On the contrary, small-scale motions are primarily responsible for the dissipation while they are weaker and contribute little to transport. From mechanical aspects, the large-scale (energy-carrying) dynamics are thus of particular importance and the costly computation of small-scale dynamics should be avoided. Furthermore, while large-scale motions are strongly dependent on the external flow conditions, small-scale motions are expected to behave more universally. Hence, there is a hope that numerical modeling can be feasible and/or require few adjustments when applied to various flows.

In LES, only the large-scale components of flow variables are explicitly integrated in time; interactions with the unresolved small-scale components are modeled. A spatial filtering is conceptually introduced as $\bar{\phi}(\mathbf{x}, t)=\int \phi\left(\mathbf{x}^{\prime}, t\right) G_{\Delta}\left(\mathbf{x}-\mathbf{x}^{\prime}\right) d \mathbf{x}^{\prime}$, where the filter width $\Delta$ fixes the size of the smallest scales of variation retained in the flow variable $\phi(\mathbf{x}, t)$ Leonard 1974). In practical applications, $\Delta$ is chosen much larger than the spatial cutoff scale of $\phi(\mathbf{x}, t)$, i.e. the dissipative scale of turbulence, so that $\bar{\phi}(\mathbf{x}, t)$ may be considered as the large-scale component of $\phi(\mathbf{x}, t)$. Applying the previous filtering procedure to the Navier-Stokes equations (and neglecting here non-commutation errors Ghosal \& Moin 1995) for the sake of simplicity) yields

$$
\frac{\partial \bar{u}_{i}}{\partial t}+\bar{u}_{j} \frac{\partial \bar{u}_{i}}{\partial x_{j}}+\frac{\partial \tau_{i j}}{\partial x_{j}}=-\frac{\partial \bar{p}}{\partial x_{i}}+\nu \frac{\partial^{2} \bar{u}_{i}}{\partial x_{k} \partial x_{k}} \quad \text { with } \quad \frac{\partial \bar{u}_{i}}{\partial x_{i}}=0
$$

where $\bar{u}_{i}(\mathbf{x}, t)$ and $\bar{p}(\mathbf{x}, t)$ represent the large-scale velocity and pressure, and $\nu$ is the kinematic viscosity of the fluid. The equations (1.1) are amenable to numerical discretisation with a grid spacing comparable to $\Delta$, since $\overline{\mathbf{u}}(\mathbf{x}, t)$ is expected to vary smoothly over the distance $\Delta . \tau_{i j}(\mathbf{x}, t) \equiv \overline{u_{i}(\mathbf{x}, t) u_{j}(\mathbf{x}, t)}-\bar{u}_{i}(\mathbf{x}, t) \bar{u}_{j}(\mathbf{x}, t)$ is named the subgrid-scale (SGS) stress tensor and encompasses all interactions between the grid-scale component and the unresolved subgrid-scale component of $\mathbf{u}(\mathbf{x}, t)$. In LES, $\tau_{i j}(\mathbf{x}, t)$ needs to be expressed in terms of the grid-scale velocity field $\overline{\mathbf{u}}(\mathbf{x}, t)$ only, which is the hard problem Lesieur 1997). Eddy-viscosity models parameterize the SGS stress tensor as

$$
\tau_{i j}-\frac{1}{3} \delta_{i j} \tau_{k k}=-2 \nu_{T} \bar{S}_{i j} \quad \text { where } \quad \bar{S}_{i j}(\mathbf{x}, t) \equiv \frac{1}{2}\left(\frac{\partial \overline{u_{i}}}{\partial x_{j}}(\mathbf{x}, t)+\frac{\partial \overline{u_{j}}}{\partial x_{i}}(\mathbf{x}, t)\right)
$$

where $\nu_{T}(\mathbf{x}, t)$ is the scalar eddy-viscosity and $\bar{S}_{i j}$ is the resolved rate-of-strain tensor. This empirical modelization is rooted in the idea that SGS motions are primarily responsible for a diffusive transport of momentum from the rapid to the slow grid-scale flow-regions. The theoretical basis for the introduction of an eddy-viscosity is rather insecure, however, it appears to be workable in practice (as advocated by Kraichnan (1976)). The eddy-viscosity $\nu_{T}$ is then primarily designed to ensure the correct mean drain of kinetic energy from the grid-scale flow to the SGS motions: $-\left\langle\tau_{i j} \bar{S}_{i j}\right\rangle$ from the equations (1.1). Another important feature is that $\nu_{T}(\mathbf{x}, t)$ should vanish in laminar flow-regions, e.g. in the viscous sub-layer near the boundary (Moin \& Kim 1982). Accordingly, our main concern was to determine an eddy-viscosity $\nu_{T}(\mathbf{x}, t)$ that would take into account shear effects in the transfer of kinetic energy to the SGS motions (without any sort of dynamical adjustment) and naturally decrease to zero at the boundary (without using any ad-hoc damping function).

In Section 2 the advantages and drawbacks of the Smagorinsky model, which is used as 
our baseline model, are recalled. The necessity for a new model that can achieve a satisfactory compromise between accuracy and manageability is identified. A shear-improved Smagorinsky model is introduced within the classical picture of shear turbulence. In Section B we present results from LES of turbulent plane-channel flows at $R e_{\tau}=395$ and $R e_{\tau}=590$. Comparisons are carried out with the dynamic Smagorinsky model and DNS. Discussion and perspectives follow in Section 4

\section{A shear-improved Smagorinsky model}

\subsection{Our baseline model: The Smagorinsky model}

The Smagorinsky model is certainly the simplest and most commonly used eddy-viscosity model (see Smagorinskv (1963), Pope (2000) for a comprehensive description). The prescription for $\nu_{T}$ writes $\nu_{T}(\mathbf{x}, t)=\left(C_{s} \Delta\right)^{2}|\bar{S}(\mathbf{x}, t)|$, where $|\bar{S}| \equiv\left(2 \bar{S}_{i j} \bar{S}_{i j}\right)^{1 / 2}$ represents the magnitude of the resolved rate-of-strain and $C_{s}$ is a non-dimensional coefficient (called the Smagorinsky constant). The major merits of the Smagorinsky model are its manageability, its computational stability and the simplicity of its formulation (involving only one adjusted parameter). All this makes it a very valuable tool for engineering applications (Rogallo \& Moin 1984). However, while this model is found to give acceptable results in the LES of homogeneous and isotropic turbulence (with $C_{s} \approx 0.17$ according to (Lilly 1967)), it is found to be too dissipative with respect to the resolved motions in near-wall turbulence, due to an excessive eddy-viscosity arising from the mean shear Moin \& Kim 1982). The eddy-viscosity predicted by Smagorinsky is non-zero in laminar flow-regions; the model introduces spurious dissipation which has the effect of damping the growth of small perturbations and thus restrain the transition to turbulence (Piomelli \& Zang 1991).

To alleviate these deficiencies in the case of wall-bounded flows, the Smagorinsky constant $C_{s}$ is often multiplied by a damping factor depending on the wall-normal distance, the van Driest function being the prime example (van Driest 1956). Although the van Driest damping function is commonly employed, its theoretical basis has never been adequately addressed, thereby leaving this function rather arbitrary. Moreover, if the determination of the distance is straightforward in the case of a plane boundary, it becomes more ambiguous near a curved boundary or a sharp corner. A more general approach, free from the use of the wall-normal distance, is therefore desirable. The dynamic SGS model intends to evaluate the Smagorinsky constant (from the resolved motions) as the calculation progresses (Germano et al. 1991) and thus avoids the need to specify a priori, or tune, its value. In brief, the adjustment of $C_{s}$ relies on the Germano identity Germano 1992) and assumes the scale similarity of resolved velocity fluctuations at scales comparable to $\Delta$ (Meneveau \& Katz 2000). This methodology yields a coefficient $C_{s}$ that varies with position and time and vanishes in the vicinity of a solid boundary with the correct behavior (Piomelli 1993). It is beyond dispute that the dynamic procedure greatly improves the capacity of the original Smagorinsky model, however, this progress is also accompanied with a certain deterioration of the numerical stability and an increase of the computational cost. In this situation, we believe that it is legitimate to seek for a SGS model that would achieve a better compromise between accuracy and manageability: ideally, as simple as the original Smagorinsky model and as accurate as the dynamic Smagorinsky model. The present SGS model that we shall call shear-improved Smagorinsky model has been elaborated along this line of idea. 


\subsection{Our proposal: A shear-improved eddy-viscosity}

Our SGS model differs from the standard Smagorinsky model in how the eddy-viscosity is defined. It borrows ideas originally advanced by Schumann (1975) (see also Sullivan et al. (1994)) by using a two-part eddy-viscosity accounting for the interplay between the nonlinear energy cascade present in isotropic turbulence, and mean shear effects associated with anisotropy. However, it differs from Schumann's proposal in its formulation, in particular for an extra simplicity. Our proposal for the SGS viscosity writes

$$
\nu_{T}=\left(C_{s} \Delta\right)^{2} \cdot(|\bar{S}|-|\langle\bar{S}\rangle|)
$$

where the brackets \langle\rangle would a priori denote an ensemble average, in practice, space average over homogeneous directions and/or time average will be used (this specific issue will be mentioned again in Section (4). From the definition (2.1) the mean drain of kinetic energy from the grid-scale to the subgrid-scale motions is given by

$$
F_{\mathrm{sgs}} \equiv-\left\langle\tau_{i j} \bar{S}_{i j}\right\rangle=\left(C_{s} \Delta\right)^{2}\left(\left\langle|\bar{S}|^{3}\right\rangle-|\langle\bar{S}\rangle|\left\langle|\bar{S}|^{2}\right\rangle\right) .
$$

Straightforwardly, our eddy-viscosity vanishes if the resolved turbulence disappears, i.e. if $\bar{S}=\langle\bar{S}\rangle$. We shall argue that it is also consistent, to some extent, with the SGS energy budget of shear turbulence. The theoretical basis of our model was first put forward by Toschi et al. (2000), on account of previous numerical and experimental studies on wallbounded turbulence (Toschi et al. 1999; Benzi et al. 1999; Ruiz-Chavarria et al. 2000). For the sake of simplicity, we shall here recast the key arguments in the ideal case of a statistically stationary homogeneous shear flow (Monin \& Yaglom 1975).

In an homogeneous shear flow, the velocity field $\mathbf{u}(\mathbf{x}, t)$ may be decomposed into $u_{i}(\mathbf{x}, t)=u_{i}^{\prime}(\mathbf{x}, t)+\left(\partial U_{i} / \partial x_{j}\right) x_{j}$, where $U_{i}(\mathbf{x})$ and $u_{i}^{\prime}(\mathbf{x}, t)$ denote the mean and fluctuating part of the velocity, respectively. Starting from the exact dynamical equations for the two-point correlation function $R(r)=\left\langle u_{i}^{\prime}(\mathbf{x}, t) u_{i}^{\prime}(\mathbf{x}+\mathbf{r}, t)\right\rangle$ and by integrating this equation over a sphere $B_{r}$ of radius $r$ centered at $\mathbf{x}$, one can establish an energy budget (at scale $r$ ) which takes the form (see Casciola et al. (2003); Danaila et al. (2004)):

$$
S_{3}^{\operatorname{tr}}(r)+S_{3}^{\mathrm{pr}}(r)=-\frac{4}{3} \varepsilon r+2 \nu \frac{\mathrm{d}}{\mathrm{d} r}\left(\frac{1}{4 \pi r^{2}} \oint_{\partial B_{r}}\left\langle\left|\delta \mathbf{u}^{\prime}(\mathbf{x}, \mathbf{r}, t)\right|^{2}\right\rangle \mathrm{dS}\right) .
$$

The two contributions $S_{3}^{\operatorname{tr}}(r)$ and $S_{3}^{\mathrm{pr}}(r)$ arise from the non-linear term of the NavierStokes equations. In the right-hand side of (2.3) $\varepsilon$ denotes the mean energy dissipation rate and the second term encompasses finite Reynolds effects (at scale $r$ ) with $\delta \mathbf{u}^{\prime}(\mathbf{x}, \mathbf{r}, t) \equiv \mathbf{u}^{\prime}(\mathbf{x}+\mathbf{r}, t)-\mathbf{u}^{\prime}(\mathbf{x}, t)$. The energy budget (2.3) is the generalization of the Karman-Howarth equation in the context of homogeneous shear turbulence (Hinze 1976). In the context of LES, it may also be interpreted as the proper mean SGS energy budget with respect to the grid scale $r$. More explicitly,

$$
S_{3}^{\operatorname{tr}}(r)=\frac{1}{4 \pi r^{2}} \oint_{\partial B_{r}}\left(\left\langle\left|\delta \mathbf{u}^{\prime}(\mathbf{x}, \mathbf{r}, t)\right|^{2} \delta u_{i}^{\prime}(\mathbf{x}, \mathbf{r}, t)\right\rangle+\frac{\partial U_{i}}{\partial x_{j}} r_{j}\left\langle\left|\delta \mathbf{u}^{\prime}(\mathbf{x}, \mathbf{r}, t)\right|^{2}\right\rangle\right) \mathrm{dS}_{i}
$$

represents the transfer of kinetic energy from grid-scale motions (at scales larger than $r$ ) to subgrid-scale motions. Also, it indicates that this transfer results from both the gridscale turbulent fluctuations and the mean shear. These two effects correspond respectively to the non-linear triple-correlation term and the rapid (or linear) term entering in the spectral decomposition of the energy transfer, as first evidenced by Crava (1958). The second term in the left-hand side of (2.3) represents a production of SGS kinetic energy 
induced by the mean shear, and is expressed as

$$
S_{3}^{\mathrm{pr}}(r)=\frac{1}{4 \pi r^{2}} \int_{B_{r}} 2 \frac{\partial U_{i}}{\partial x_{j}}\left\langle\delta u_{i}^{\prime}(\mathbf{x}, \mathbf{r}, t) \delta u_{j}^{\prime}(\mathbf{x}, \mathbf{r}, t)\right\rangle \mathrm{dV} .
$$

In summary, the energy budget 2.3) means that in the steady-state, the SGS dynamics are sustained against molecular dissipation (represented by the right-hand side of (2.3)) by the transfer of energy originated from grid-scale motions $\left(S_{3}^{\operatorname{tr}}(r)\right)$ and the production of energy induced directly by the mean shear $\left(S_{3}^{\mathrm{pr}}(r)\right)$.

As mentioned in the introduction, the estimate of the SGS energy flux, which should be identified with $S_{3}^{\operatorname{tr}}(\Delta) / \Delta$ in the previous developments, is of prime importance in the modeling of the eddy-viscosity. Roughly speaking, one may consider that turbulent gridscale velocity differences $\delta u^{\prime}(\Delta)$ typically behave as the fluctuating part of the resolved rate-of-strain $\left|\overline{S^{\prime}}\right|$ multiplied by $\Delta$, i.e. $\delta u^{\prime}(\Delta) \approx\left|\overline{S^{\prime}}\right| \Delta$. In the same way, $\delta U(\Delta) \approx|\langle\bar{S}\rangle| \Delta$. Equation (2.4) then suggests that the mean SGS energy flux should involve two separate contributions of order $\Delta^{2}\left\langle\left|\overline{S^{\prime}}\right|^{3}\right\rangle$ and $\Delta^{2}|\langle\bar{S}\rangle|\left\langle\left|\overline{S^{\prime}}\right|^{2}\right\rangle$, respectively.

In flow-regions where $\left|\overline{S^{\prime}}\right| \gg|\langle\bar{S}\rangle|$, the SGS energy flux reduces to the contribution of order $\Delta^{2}\left\langle\left|\bar{S}^{\prime}\right|^{3}\right\rangle$. In these regions, the mean shear is too weak to perturb the grid-scale dynamics; eddies of size comparable to the grid-scale $\Delta$ adjust dynamically via nonlinear interactions to transfer energy to SGS motions. This is the standard mechanism behind homogeneous and isotropic turbulence (Frisch 1995). From our expression (2.2), we remark that the Smagorinsky estimate $F_{\mathrm{sgs}} \simeq\left(C_{s} \Delta\right)^{2}\left\langle\left.\overline{S^{\prime}}\right|^{3}\right\rangle$ is consistently recovered in that case. In regions where $|\langle\bar{S}\rangle| \gg\left|\overline{S^{\prime}}\right|$, the behavior of the flow is clearly different: eddies of size comparable to the grid-scale have no time to adjust dynamically and are rapidly distorted by the mean shear (Liu et al. 1999). In these regions, the SGS energy flux is driven by the mean shear and therefore is dominated by the contribution of order $\Delta^{2}|\langle\bar{S}\rangle|\left\langle\left|\overline{S^{\prime}}\right|^{2}\right\rangle$. From expression (2.2) and assuming that $\left\langle|\bar{S}|^{3}\right\rangle \approx\left\langle|\bar{S}|^{2}\right\rangle^{3 / 2}$, one obtains $F_{\text {sgs }} \simeq 1 / 2\left(C_{s} \Delta\right)^{2}|\langle\bar{S}\rangle|\left\langle\mid{\overline{S^{\prime}}}^{2}\right\rangle$ in agreement with the previous reasoning. We may thus conclude that proposal (2.1) for the eddy-viscosity is consistent with the SGS energy budget of (locally homogeneous) shear turbulence in the two limiting situations $\left|\overline{S^{\prime}}\right| \gg|\langle\bar{S}\rangle|$ and $|\langle\bar{S}\rangle| \gg\left|\overline{S^{\prime}}\right|$. The first results concerning plane-channel flows (see Section 3indicate that the proposed model abridges between these two situations without the need for any additional adjustment.

\section{Les of turbulent plane-channel flows}

Over the last twenty years LES of wall-bounded flows have received a considerable attention (see Piomelli \& Balaras (2002) for a recent review), with the turbulent planechannel flow (Kim et al. (1987) for an original work) being the prototypical case. This flow allows for the investigation of shear effects in a simple geometry and has therefore provided a useful test bed of our eddy-viscosity model. Furthermore, we have been able to confront our results, including mean velocity, turbulence intensities and Reynolds stress profiles, with the well-established literature present on that case, e.g. the comprehensive DNs database obtained by Moser et al. (1999) or, more recently, by Hovas \& Jimenez (2006).

\subsection{Numerical simulations}

We performed two LES at $R e_{\tau}=395$ and $R e_{\tau}=590$, where $R e_{\tau}$ is the Reynolds number based on the friction velocity $u_{\tau}: R e_{\tau} \equiv u_{\tau} H / \nu$ ( $H$ is the half width of the channel). These two cases correspond to the DNs conducted by Moser et al. (1999). Periodic boundary conditions were imposed in the streamwise and spanwise directions; 


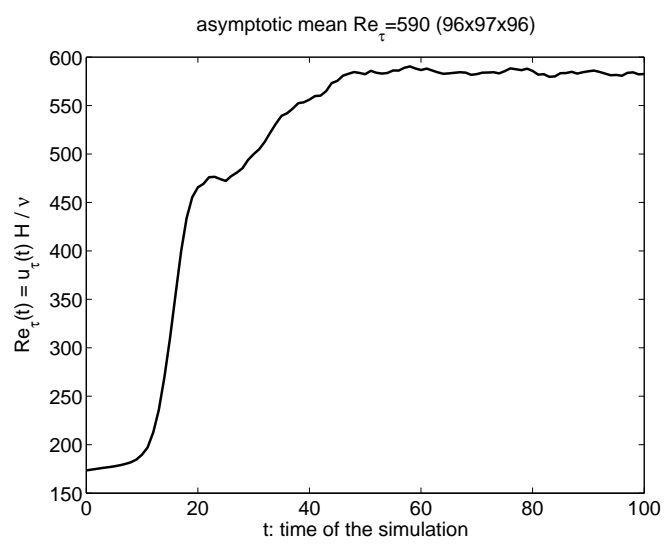

Figure 1. The time development of the Reynolds number $R e_{\tau}(t)$ based on the plane-averaged friction velocity $u_{\tau}(t)$.

no-slip conditions at the wall. The flow was simulated by integrating the filtered NavierStokes equations (1.1) with the prescribed eddy-viscosity 2.1). The right-hand side of the equations (1.1) was supplemented by an (external) pressure-gradient $\Delta p_{\text {ext }} / 4 \pi H$ in order to drive the flow in the streamwise direction. The pressure difference $\Delta p_{\text {ext }}(t)$ was adjusted dynamically to keep a constant flow rate through the channel. The integration in time relied on a Fourier-Chebyshev pseudo-spectral solver (de-aliased by using the $3 / 2$ rule) based on a third-order Runge-Kutta scheme. More details about the numerical simulations can be found in Xu et al. (1996). The Smagorinsky constant was fixed to its standard value $C_{s}=0.16$ (in the case of homogeneous and isotropic turbulence) and the scale $\Delta$ was estimated as $(\Delta x \Delta y \Delta z)^{1 / 3}$, where $\Delta x, \Delta y$ and $\Delta z$ denote the local grid sizes in each direction. In the following, the grid-scale velocity components are $U+u^{\prime}, v^{\prime}$ and $w^{\prime}$ along the streamwise, wall-normal and spanwise directions, respectively.

\subsection{Numerical results}

At initial time, velocity distributions were designed to satisfy the Poiseuille profile plus a small random perturbation. The time development of $R e_{\tau}(t)=u_{\tau}(t) H / \nu$, where $u_{\tau}(t)$ expresses as the square root of the plane-averaged wall shear stress, is plotted in Figure [1 for the case $R e_{\tau}=590$. A transition (or drag crisis) to the appropriate turbulent regime occurs naturally as the integration (involving the eddy-viscosity (2.1)) progresses. This feature constitutes a first improvement over the Smagorinsky model, for which such transition is not captured. Once a developed turbulent state was achieved, statistics were accumulated.

The mean velocity $U^{+}\left(y^{+}\right)$is displayed as a function of the wall-normal distance $y^{+}$in Figure 2 From now on, the average is meant in time and over horizontal $x-z$ planes (homogeneous directions). Mean velocity profiles agree very well with the DNS data of reference obtained by Moser et al. (1999) for both considered Reynolds numbers. The comparison with the dynamic Smagorinsky model is also very satisfactory (Piomelli 1993). Turbulence intensity profiles (normalized by the squared friction velocity) are displayed in Figure 3 Also here, the profiles compare very well with the DNS data and LES results based on the dynamic Smagorinsky model: notice how peak values are suitably captured. As for the dynamic Smagorinsky model, a slight drop is observed for the spanwise and wall-normal components, in the log-layer; on the contrary, the streamwise component fits remarkably the DNS data. In the proximity of the wall, the correct behavior is obtained. 

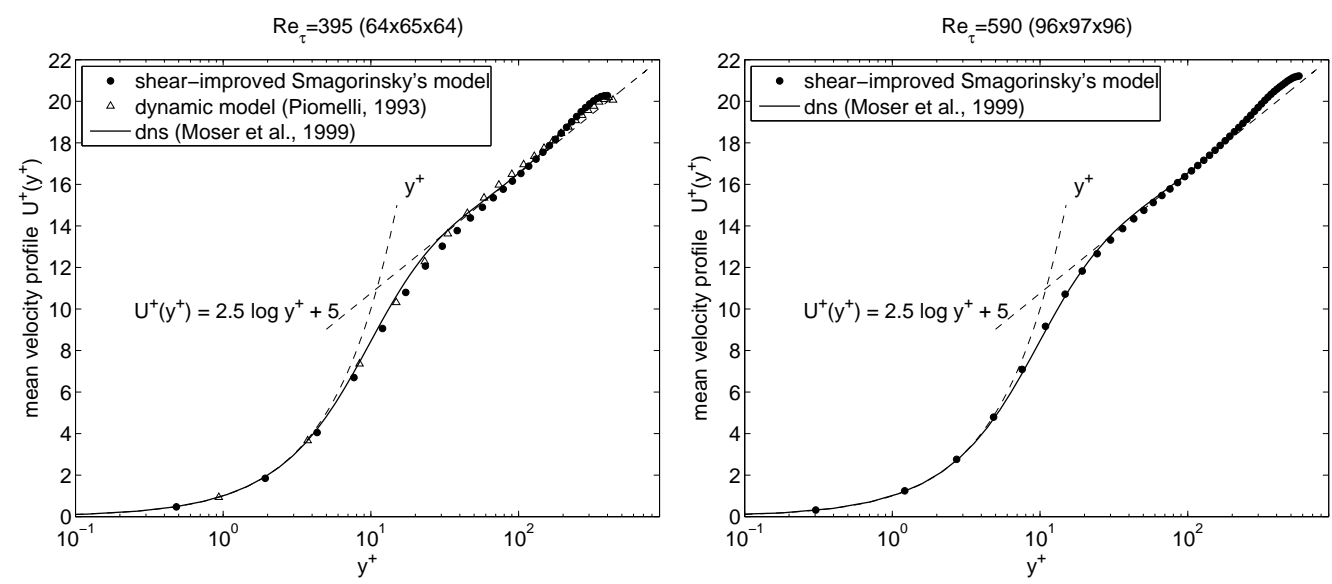

Figure 2. Left: $(\bullet)$ mean-velocity profile (in wall units) at $R e_{\tau}=395$. The computational domain (in outer units) is $4 \pi H \times 2 H \times 2 \pi H$ with $64 \times 65 \times 64$ grid points. In comparison with (-) the DNs data obtained by Moser et al. (1999) in the domain $2 \pi H \times 2 H \times \pi H$ with $256 \times 193 \times 192$ grid points, and $(\triangle)$ a computation of the dynamic Smagorinsky model carried out by Piomelli (private communication) in the domain $5 \pi H / 2 \times 2 H \times \pi H / 2$ with $48 \times 49 \times 48$ grid points (using a pseudo-spectral solver). Right: $(\bullet)$ mean-velocity profile at $R e_{\tau}=590$ with $96 \times 97 \times 96$ grid points. In comparisons with $(-)$ the DNs data with $384 \times 257 \times 384$ grid points.
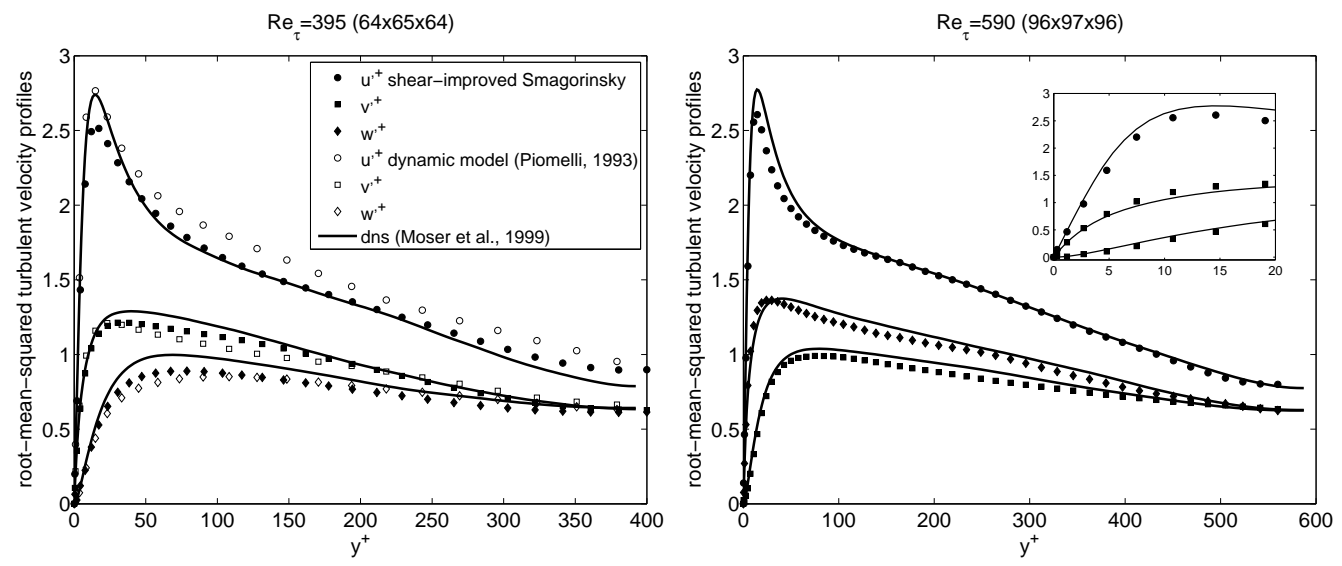

FiguRE 3. Left: Turbulent intensity profiles at $R e_{\tau}=395$ in comparison with DNs data (-) and LES data obtained with the dynamic Smagorinsky SGS model (open symbols). Right: Turbulent intensity profiles at $R e_{\tau}=590$. The inset focuses on the near-wall behavior in comparison with DNs data.

These positive results make us presume that our eddy-viscosity has captured the essential of shear effects in wall-bounded turbulence. Now, in order to better check this hypothesis, the ratio $\sqrt{\left\langle\left|S^{\prime}\right|^{2}\right\rangle} /|\langle S\rangle|$ is shown as a function of the wall-normal distance in Figure 4 For $y^{+} \lesssim 25$, one can see that the mean shear dominates over the fluctuating part of the rate-of-strain, indicating the predominance of the mean-shear component of the eddy-viscosity in that region. Note that the transition distance $y^{+} \simeq 25$ is fully consistent with the empirical characteristic distance $A^{+}=25$ commonly used in the van Driest damping function (Pope 2000). In the log-layer, $\sqrt{\left\langle\left. S^{\prime}\right|^{2}\right\rangle} /|\langle S\rangle|$ increases slowly with $y^{+}$(the standard description of the log-layer predicts a linear increase, according to $|\langle S\rangle| \propto 1 / y$ and $\left\langle\left|S^{\prime}\right|^{2}\right\rangle \propto u_{\tau}^{2} / \Delta^{2}$ (Schlichting 1955) ) and eventually diverges around the 


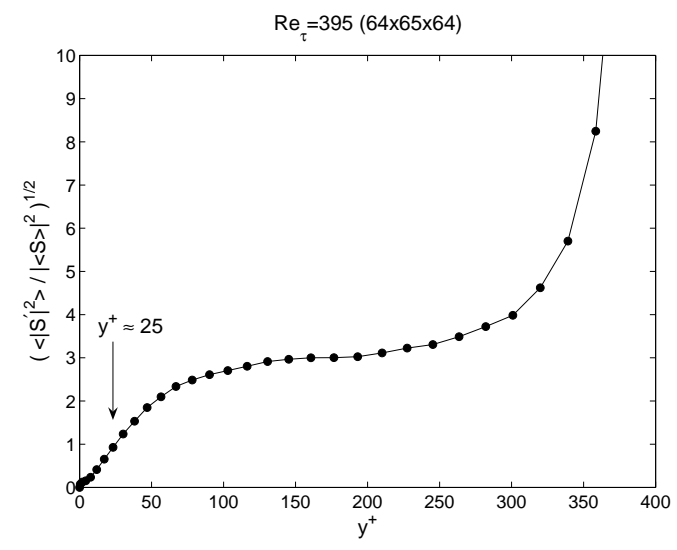

Figure 4. The ratio $\sqrt{\left\langle\left|S^{\prime}\right|^{2}\right\rangle} /|\langle S\rangle|$ is displayed as a function of the wall-normal distance $y^{+}$ in the LES at $R e_{\tau}=395$. For $y^{+} \lesssim 25$, the mean shear dominates over the fluctuating part of rate-of-strain. In that region, our eddy-viscosity differs from the original Smagorinsky model candidate.
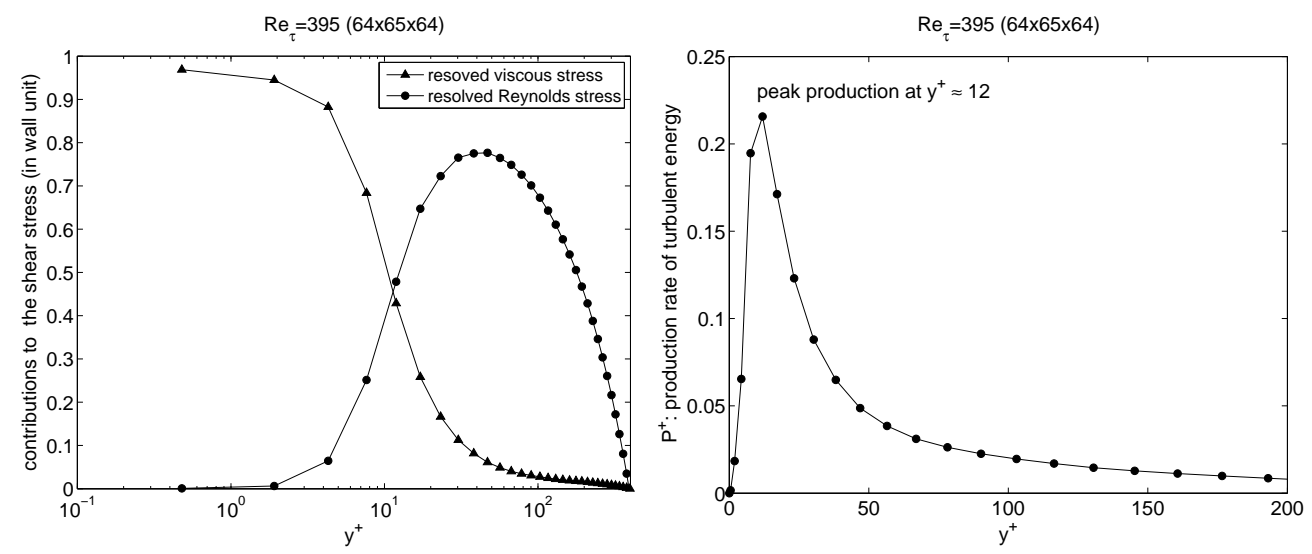

FiguRE 5. Left: The viscous and Reynolds stresses (computed from the resolved velocity) at $R e_{\tau}=395$. The two contributions are equal at $y^{+} \approx 12$, in full agreement with DNs results. Right: The peak production of turbulent energy also occurs at $y^{+} \approx 12$, as expected.

centerline of the channel. Thus, we may claim that our model suitably abridges between the situation where the mean shear prevails (close to the boundaries) and the situation where the fluctuating part of the rate-of-strain dominates (in the bulk of the channel).

The viscous, $d U^{+} / d y^{+}$, and the Reynolds, $\left\langle u^{+} v^{+}\right\rangle$, contributions to the resolved stress are shown in Figure 5 . They equal for $y^{+} \approx 12$ in perfect agreement with DNs results (Pope 2000). The peak value of the turbulent energy production, $\left\langle u^{+} v^{+}\right\rangle d U^{+} / d y^{+}$, occurs at the same distance $y^{+} \approx 12$, as expected theoretically from the Navier-Stokes equations. The grid-scale Reynolds stress profile is displayed in Figure 6 The agreement with the DNS and the LES based on the dynamic Smagorinsky model is fair and also the behavior close to the wall is very satisfactory. 

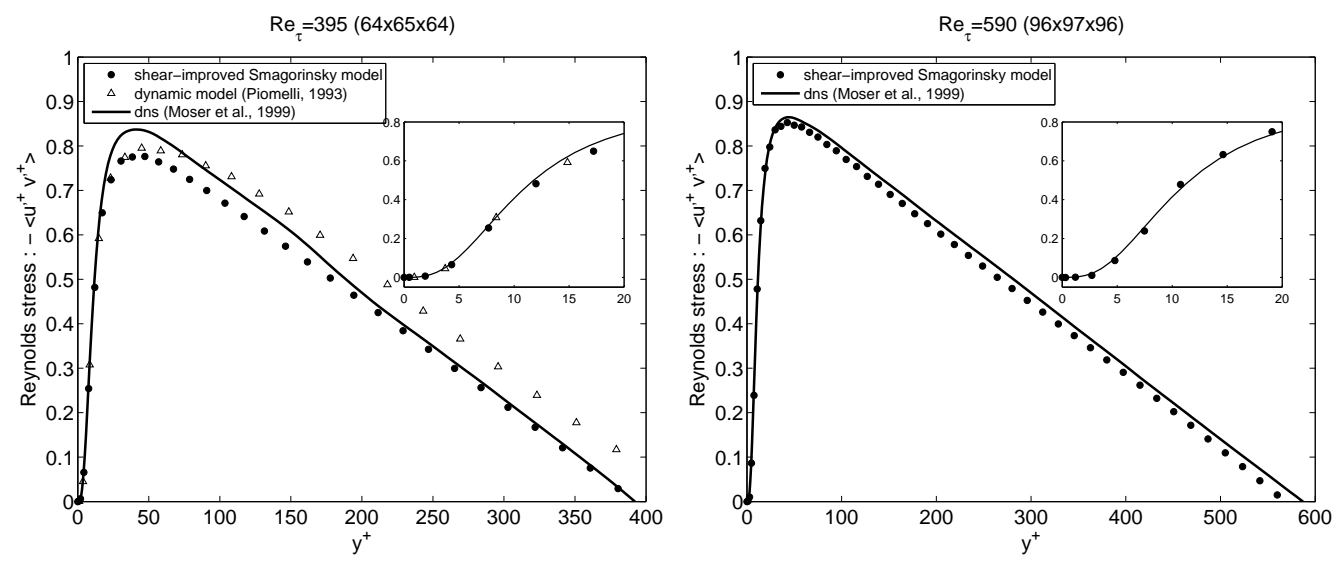

FiguRE 6. Left: Reynolds stress at $R e_{\tau}=395$ (computed from the resolved velocity). Right: The Reynolds stress at $R e_{\tau}=590$. The insets focus on the near-wall behavior.

\section{Discussions and perspectives}

Concluding, we presented a very simple SGS model consisting in a physically sound improvement over the well-established Smagorinsky model. An explicit connection with the scale-by-scale energy budget of homogeneous shear turbulence has also been identified. Our first results for turbulent plane-channel flows indicate that the proposed model possesses a very good predictive capability (essentially equivalent to the dynamic Smagorinsky model) with a computational cost and a manageability comparable to the original Smagorinsky model.

The generalization to more complex non-homogeneous flows is a priori straightforward since no geometrical argument enters in the definition of the eddy-viscosity. However, an appropriate average must be specified in the absence of homogeneity directions. As a natural candidate, we suggest an average in time to evaluate the mean components of the rate-of-strain tensor (assuming that the flow is statistically stationary). In the case of non-stationary flows, an ensemble average (over several realizations of the flow) may be envisaged. These points are the subject of current investigations in progress.

The authors are grateful to Ugo Piomelli for providing numerical data; in particular F.T. wishes to thank him for the many useful discussions. Fang Le helped us to perform the LES at the Tsinghua University in China. This work was jointly supported by the École normale supérieure de Lyon and the École centrale de Lyon under a Bonus-QualitéRecherche grant. Finally, F.T. acknowledges E.L. for his kind hospitality at the EnsLYON.

\section{REFERENCES}

Benzi, R., Amati, G., Casciola, C., Toschi, F. \& Piva, R. 1999 Intermittency and scaling laws for wall bounded turbulence. Physics of Fluids 11 (6), 1284-1286.

Casciola, C. M., Gualtieri, P., Benzi, R. \& Piva, R. 2003 Scale-by-scale budget and similarity laws for shear turbulence. J. Fluid Mech. 176, 105-114.

Craya, A. 1958 Contribution à l'analyse de la turbulence associée à des vitesses moyennes. $P$. S. T. Ministère de l'Air $\mathbf{3 4 5}$.

Danaila, L., Antonia, R. \& Burattini, P. 2004 Progress in studying small-scale turbulence using exact two-point equations. New J. Physics 6, 128. 
DEARdorfF, J. W. 1970 A numerical study of three-dimensional turbulent channel flow at large reynolds numbers. J. Fluid Mech. 41, 453.

VAn Driest, E. R. 1956 On turbulent flow near a wall. J. Aero. Sci. 23, 1007-1011.

Frisch, U. 1995 Turbulence: The legacy of A. N. Kolmogorov. Cambridge University Press.

Germano, M. 1992 Turbulence: the filtering approach. J. Fluid Mech. 238 (325-36).

Germano, M., Piomelli, U., Moin, P. \& Cabot, W. H. 1991 A dynamic subgrid-scale eddy viscosity model. Phys. Fluids A 3, 1760.

Ghosal, S. \& Moin, P. 1995 The basic equations for the large eddy simulation of turbulent ows in complex geometry. J. Comp. Phys. 118 (1), 24-37.

Hinze, J. O. 1976 Turbulence. McGraw-Hill.

Hoyas, S. \& Jimenez, J. 2006 Scaling of velocity fluctuations in turbulent channels up to $r e_{\tau}=2000$. Phys. of Fluids 18, 011702, http://torroja.dmt.upm.es/ftp/channels/data/.

Kim, J., Moin, P. \& Moser, R. D. 1987 Turbulent statistics in fully developed channel flow at low reynolds number. J. Fluid Mech. 177, 133.

Kraichnan, R. H. 1976 eddy-viscosity in two and three dimensions. J. Atmos. Sci. 33, 15211536.

LEONARD 1974 Energy cascade in large-eddy simulations of turbulent fluid flows. Adv. in Geophys. A 18, 237-248.

Lesieur, M. 1997 Turbulence in Fluids, 3rd edn. Dordrecht: Kluwer.

Lesieur, M. \& Metais, O. 1996 New trends in large-eddy simulations of turbulence. Ann. Rev. Fluid. Mech. 28, 45-82.

LiLly, D. K. 1967 The representation of small-scale turbulence in numerical simulation experiments. In Proc. IBM Scientific Computing Symp. Environ. Sci., p. 195.

Liu, S., Katz, J. \& Meneveau, C. 1999 Evolution and modelling of subgrid scales during rapid straining of turbulence. J. Fluid Mech. 387, 281-320.

Meneveau, C. \& Katz, J. 2000 Scale-invariance and turbulence models for large-eddy simulation. Annu. Rev. Fluid Mech. 32, 1-32.

Moin, P. \& Kim, J. 1982 Numerical investigation of turbulent channel flow. J. Fluid Mech. 118, 341-377.

Monin, A. S. \& Yaglom, A. M. 1975 Statistical Fluid Mechanics, , vol. 2. MIT Press, Cambridge.

Moser, R. D., Kim, J. \& Mansour, N. N. 1999 Direct numerical simulation of turbulent channel flow up to $r e_{\tau}=$ 590. Physics of Fluids 11 (4), 943-945, http://www.tam.uiuc.edu/Faculty/Moser.

Perot, B. \& Moin, P. 1995 Shear-free turbulent boundary layers, part i: Physical insights into near wall turbulence. J. Fluid Mech. 295, 199.

Piomelli, U. 1993 High reynolds number calculations using the dynamic subgrid-scale stress model. Phys. Fluids A 5 (6), 1484.

Piomelli, U. 1999 Large-eddy simulation: Achievements and challenges. Progress in Aerospace Sciences 35, 335-362.

Piomelli, U. \& Balaras, E. 2002 Wall-layer models for large-eddy simulations. Annu. Rev. Fluid Mech. 34, 349-374.

Piomelli, U. \& ZAng, T. A. 1991 Large-eddy simulation of transitional channel flow. Comput. Phys. Commun. 65, 224-30.

Pope, S. B. 2000 Turbulent flows. Cambridge University Press.

Rogallo, R. S. \& Moin, P. 1984 Numerical simulation of turbulent flows. Annu. Rev. Fluid Mech. 16, 99-137.

Ruiz-Chavarria, G., Ciliberto, S., Baudet, C. \& Lévêque, E. 2000 Scaling properties of the streamwise component of velocity in a turbulent boundary layer. Physica $D$ 141, $183-198$.

Sagaut, P. 2001 Large Eddy Simulation for Incompressible Flows. Springer.

Schlichting, H. 1955 Boundary-layer theory. McGraw-Hill.

Schumann, U. 1975 Subgrid scale model for finite difference simulations of turbulent flows in plane channels and annulli. J. Comput. Phys. 18, 376-404.

Smagorinsky, J. 1963 General circulation experiments with the primitive equations. i. the basic experiment. Mon. Weather Rev. 91, 99. 
Sullivan, P., McWilliams, J. C. \& Moeng, C.-H. 1994 A subgrid-scale model for large-eddy simulation of planetary boundary-layer flows. Bound.-Layer Meteorol. 71, 247-76.

Toschi, F., Amati, G., Succi, S., Benzi, R. \& Piva, R. 1999 Intermittency and structure functions in channel flow turbulence. Physical Review Letters 82 (25), 5044-5047.

Toschi, F., Lévêque, E. \& Ruiz-Chavarria, G. 2000 Shear effects in nonhomogeneous turbulence. Phys. Rev. Lett. 85 (7), 1436.

Xu, C. X., Zhang, Z. \& Nieuwstatd, F. M. 1996 Origin of high kurtosis in viscous sublayer. Phys. Fluids 85, 1938. 